\title{
Spatial distribution of caatinga plants in the environment of a thermelectric
}

The vegetation of the Caatinga of the Brazilian semiarid of the xerophilous type is usually found between shallow and poorly permeable soils, which is constantly affected by irregular deforestation. In this context, the objective of this work was to identify the floristic species of a thermoelectric plant located in the semiarid Paraibano region, in addition to diagnosing the ecological integrity of the arboreal remnants of this area. The Phytosociological Survey of this study was carried out in an area with 20.1 ha, with sampling in six plots. The processing of phytosociological data was carried out using Mata Nativa 4.0 software. The identification of floristic species was evaluated through the basal area, density, frequency, dominance, cover value and importance value. For the analysis of the data, the similarity between the species was compared by the Jaccard index, the floristic similarity matrix was used for cluster analysis and dendrogram generation. The floristic species inventoried, totaled 280 individuals and the family that had the largest representative was the Euphorbiaceae. It was observed that the studied area is in process of ecological succession, due to the physical pressures of the residents of the surroundings, with exploitation of natural resources of uncontrolled form causing, deforestation, burnings, among others. It is verified that floristic inventories are of great importance to monitor biodiversity, as well as to draw up, goals of efficient environmental management and serve as a tool aimed at the conservation of the Caatinga species.

Keyword: Phytosociological Survey; Biodiversity; Xerophilous Vegetation

\section{Distribuição espacial de plantas de caatinga no ambiente de uma termelétrica}

\begin{abstract}
A vegetação da Caatinga do semiárido brasileiro do tipo xerófila é geralmente encontrada entre solos rasos e pouco permeáveis, que é constantemente afetado pelo desmatamento irregular. Neste contexto, o objetivo deste trabalho foi identificar as espécies florísticas de uma usina termoelétrica localizada na região semiárida do Paraibano, além de diagnosticar a integridade ecológica dos remanescentes arbóreos dessa área. O Levantamento Fitossociológico deste estudo foi realizado em uma área com 20,1 ha, com amostragem em seis parcelas. O processamento dos dados fitossociológicos foi realizado utilizando o software Mata Nativa 4.0. A identificação das espécies florísticas foi avaliada através da área basal, densidade, freqüência, dominância, valor de cobertura e valor de importância. Para a análise dos dados, a similaridade entre as espécies foi comparada pelo índice de Jaccard, a matriz de similaridade florística foi utilizada para análise de agrupamento e geração de dendrogramas. As espécies florísticas inventariadas totalizaram 280 indivíduos e a família que apresentou maior representatividade foi a Euphorbiaceae. Observou-se que a área estudada está em processo de sucessão ecológica, devido às pressões físicas dos moradores do entorno, com exploração de recursos naturais de forma descontrolada causando, desmatamento, queimadas, entre outros. Verifica-se que os inventários florísticos são de grande importância para o monitoramento da biodiversidade, assim como para a elaboração de metas de manejo ambiental eficiente e servem como ferramenta para a conservação das espécies da Caatinga.
\end{abstract}

Palavras-chave: Levantamento Fitossociológico; Biodiversidade; Vegetação Xerófila.

Topic: Ciências Florestais

Reviewed anonymously in the process of blind peer
Received: 10/02/2018

Approved: $24 / 03 / 2018$
Joaci dos Santos Cerqueira (iD)

Universidade Federal de Campina Grande, Brasil

http://lattes.cnpq.br/4470582307237189

http://orcid.org/0000-0002-0109-3849

cerq2006@gmail.com

Helder Neves de Albuquerque

Universidade Federal de Campina Grande, Brasil

http://lattes.cnpq.br/1316763030688970

http://orcid.org/0000-0002-5076-3060

helderbiologo@gmail.com

Francisco de Assis Salviano de Sousa (iD

Universidade Federal de Campina Grande, Brasil

http://lattes.cnpq.br/5392432872592612

http://orcid.org/0000-0002-4085-0785

fsouza2011@gmail.com
Referencing this:

CERQUEIRA, J. S.; ALBUQUERQUE, H. N.; SOUSA, F. A. S.. Spatial distribution of caatinga plants in the environment of a thermelectric. Revista Ibero Americana de Ciências Ambientais, v.9, n.3, p.1-11, 2018. DOI: http://doi.org/10.6008/CBPC2179-6858.2018.003.0001 


\section{INTRODUCCION}

The Northeast region of Brazil covers an area equivalent to $18 \%$ of the national territory. Since the beginning of colonization its occupation has been subjected to uncontrolled and illogical deforestation. Initially, the Mata Atlântica was suppressed for the implantation of cane plantations on the coast and, with the implantation of extensive cattle raising and expansion of agriculture, new areas were sought in the interior, where a great part of the Caatinga biome was destroyed and changed (BESSA et al., 2011).

The Caatinga can be characterized as having a vegetation of the xerophilous type, of the Brazilian semi - arid regions, specifically, found on the shallow and slightly permeable soils of the crystalline outcroppings, widely distributed in the region. Thus, the northeastern wilderness should be considered as part of the caatingas, as a hypoxerophilic variant of the communities found to the west of them (OLIVEIRA et al., 2003).

High deforestation in the Caatinga Biome has led to desertification processes in several areas, thus altering the biota, microclimate and soils, and it is essential to develop research techniques capable of incorporating information that identifies the state of natural resources, pointing out their relationships and actions to be taken for an efficient intervention that will lead to the recovery and sustainable use of soils and vegetation in this environment (SOUZA et al., 2015).

Other anthropogenic effects such as air pollution damage significantly affect semiarid flora. Plants are beings that respond faster to this type of pollution, which are useful for establishing the first air pollutants, determining the geographic distribution of pollutants, estimating the concentration of pollutants and making it possible to identify the different atmospheric pollutants, depending on the variety of the plants affected, making them excellent bioindicators (CEN, 2015; MORAES et al., 2011).

Environmental factors such as soil, geographic aspect and temperature, in addition to precipitation, play an important role in the persistence of plants and, consequently, contribute to the biological diversity of a particular location. The composition, structure and vegetative functions of the surrounding species are also very critical ecological attributes, indicating large variations in response to the environment. It is also observed that the formation of clearings and rings in plants, make up a specific pattern as an environmental response (light, temperature, drought, soil properties, wind direction and / or anthropogenic causes) (TARDIEU, 2013).

Vegetation is of great environmental importance because it controls air pollution and erosion. Besides protecting slopes from landslides it is essential for the feeding, sheltering, nesting and breeding of animals. Among the factors responsible for the degradation of forest areas are the exploitation of unsustainable forest products, overgrazing, fires and pollution. As a result, forest cover in most tropical countries has declined at an alarming rate in recent decades (PATRA et al., 2015).

Hence the need for biodiversity conservation to have gained widespread acceptance across much of the planet due to overcoming the conflict between the preservation and use of nature, which advocates for the importance of current protection in the name of potential benefit. This acceptance gains strength as 
science discovers new uses for plants and animals hitherto uninteresting and as the areas where these species occur have been altered (GIULIETTI et al., 2004). In this context, the objective of this work was to identify the floristic species around a thermoelectric plant located in the semiarid Paraiba region, in addition to diagnosing the ecological integrity of the arboreal remnants of this area.

\section{MATERIALS AND METHODS}

\section{Location and characterization of the study area}

The study was carried out in an area with tree / shrub physiognomy, presenting floristic remnants of the semiarid region, located in the surroundings of the Borborema Energética S.A Thermoelectric Plant. An area of 20.1ha was used in this study (Figure 1), plots being randomly chosen in relation to the distances of $100,200,300,400,500$ and $1000 \mathrm{~m}$. The area has flat relief and, average altitude of $487 \mathrm{~m}$, and the soils are shallow and stony, predominantly of the types panosols and vertisols. Therefore, the vegetation is characterized as a hypoxerophilic caatinga, typical of the Brazilian semiarid region, which presents traces of vegetation from the Caatinga/Mata Atlântica ecotone (SANTOS et al., 2006).

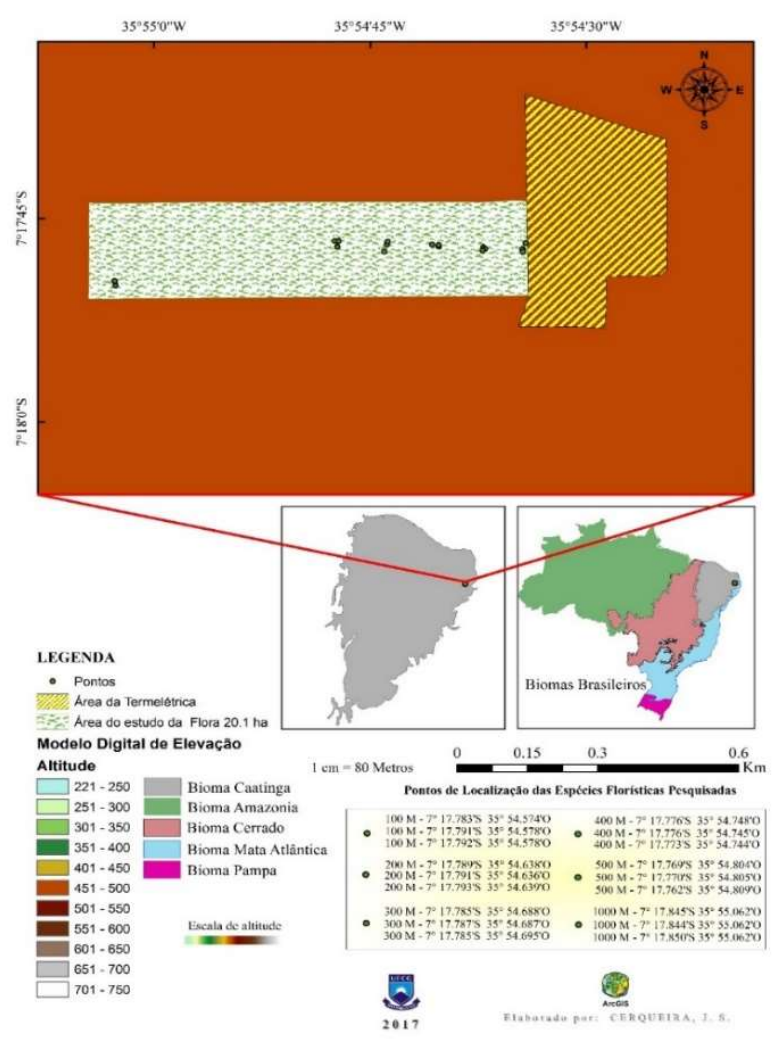

Figure 1: Location of floristic study area with elevation points.

\section{Flora survey}

The research was carried out from January to December 2016 and for the Phytosociological Survey (LF), sampling of the shrub-tree component was performed according to the method adopted by MullerDombois et al. (1974) and Rodal (2006), through the location of 6 contiguous plots of $10 \mathrm{~m} \times 10 \mathrm{~m}$, in each area. Geoprocessing tools used were ArcMap 10.1 (2011) and GPS. Sampling points were pre-defined, where 
it was sought to inventory the sites that could present greater arboreal representativeness, thus avoiding clearings and the edges of the study area.

As a technique for measuring floristic species, dendrometry was used to determine the volume of species most efficiently (OLIVEIRA, 2014). To measure plant height, the range finder can be used. However, in the absence of one, it is possible to use graduated rods or embedded PVC pipes (MORO et al., 2011). In this way, the height of the plants was identified through PVC rods of $2 \mathrm{~m}$ height, fitted and placed close to the plant to be measured. The PVC pipes also received a marking every 10, 25 or $50 \mathrm{~cm}$ to detail the height of the plants. The floristic list was organized according to the APG IV System (2016) and the list of authors of species, according to Brummitt et al. (1992).

The processing of phytosociological data was carried out using Mata Nativa 4.0 software (CIENTEC, 2016). As research material, we used pachymeter, tape measure, PVC pipe, annotation block, GPS Garmin Gpsmap 62sc 5mp camera, semi-professional Canon powershot SX60HS 16.1MP LCD 3.0 digital camera, optical zoom 65x, microcomputer, computer network and peripherals and software (Excel 2016, ArcMap 2011, licensed to the Laboratory of Digital Cartography, Geoprocessing and Remote Sensing - CADIGEOS, of the graduate courses of the Humanities Center of the Federal University of Campina Grande - UFCG).

The similarity between species was also compared by the Jaccard index, which expresses the similarity between environments, based on the number of common species. The resulting floristic similarity matrix was used for group analysis by the unweighted arithmetic means (UPGMA) method and by the generation of a dendrogram (SNEATH et al., 1973).

\section{Floristic and structural composition}

The identification of the floristic species in the study area was standardized according to the diameter at breast height (CAP), height and number of tillers. The vegetation structure was evaluated through basal area, density, frequency, dominance, cover value and importance value (BROWN-BLANQUET, 1979; MÜLLERDOMBOIS et al., 1974; KENT et al., 1999).

\section{RESULTS}

In the area surveyed, it was verified that the floristic species, totaled 280 individuals, distributed in 17 families and 23 different species. The family that had the highest representation was Euphorbiaceae $(n=4)$ and species that had only one representative were, Jatropha curcas, Tabebuia aurea, Clitorea ternatea, Capparis flexuosa L., Libidibia ferrous, Pisonia tomentosa, Schinopsis brasiliensis and Ceiba glaziovii. Thus, it is evident that the sample area presented a diversified number of families and a small number of individuals per plot.

In table 1, it is shown that the occurrence of greater richness was Combretum glaucocarpum ( $\mathrm{N}=61$ ). The species that had the greatest diversity was the Croton sonderianus, with specimens in all plots. It was followed consecutively by the species Aspidosperma pyrifolium, Mimosa Tenuiflora and Piptdenia stipulacea, all found in 5 plots. It was also observed that the species Jatropha curcas, Tabebuia aurea, Clitorea ternatea, 
Capparis flexuosa L., Libidibia férrea, Pisonia tomentosa, Schinopsis brasiliensis, and Ceiba glaziovii occurred in only one of the plots and had a single representative $(\mathrm{N}=1)$.

Table 1: List of floristic species represented by richness, percentage and distribution in plots.

\begin{tabular}{|l|l|l|l|l|}
\hline Code & Scientific name & $\mathbf{N}$ & $\%$ & Parcels \\
\hline 1 & Croton sonderianus & 50 & 17.86 & $1,2,3,4,5,6$ \\
\hline 2 & Aspidosperma pyrifolium & 44 & 15.71 & $1,2,3,4,5$ \\
\hline 3 & Mimosa tenuiflora Wild & 32 & 11.43 & $1,2,4,5,6$ \\
\hline 4 & Piptdenia stipulacea Benth & 23 & 7.5 & $1,2,3,4,5$ \\
\hline 5 & Poincianella pyramidalis Tul. & 12 & 4.29 & $2,3,4,5$ \\
\hline 6 & Uncaria tomentosa & 5 & 1.79 & $1,2,3$ \\
\hline 7 & Combretum glaucocarpum & 61 & 21.78 & $2,3,4$ \\
\hline 8 & Bauhinia forficata Linn & 25 & 8.93 & $2,4,5$ \\
\hline 9 & Acacia glomerosa Benth & 2 & 0.71 & 2,3 \\
\hline 10 & Capparis yco & 3 & 1.07 & 2,4 \\
\hline 11 & Manihot pseudoglaziovii & 4 & 1.43 & 4,5 \\
\hline 12 & Jatropha curcas & 1 & 1.07 & 1 \\
\hline 13 & Croton heliotropiifolius & 3 & 1.07 & 1 \\
\hline 14 & Tabebuia aurea & 1 & 0.36 & 2 \\
\hline 15 & Clitorea ternatea & 1 & 0.36 & 2 \\
\hline 16 & Capparis flexuosa L. & 1 & 0.36 & 2 \\
\hline 17 & Libidibia ferrea & 1 & 0.36 & 2 \\
\hline 18 & Pisonia tomentosa & 1 & 0.36 & 3 \\
\hline 19 & Commiphora leptophloeos & 3 & 1.07 & 3 \\
\hline 20 & Schinopsis brasiliensis & 1 & 0.36 & 4 \\
\hline 21 & Eugenia pyriformis var. uvalha & 2 & 0.71 & 5 \\
\hline 22 & Ceiba glaziovii & 1 & 0.36 & 5 \\
\hline 23 & Ziziphus joazeiro & 3 & 1.07 & 6 \\
\hline
\end{tabular}

Legend: $\mathrm{N}$ = richness; \% = percentage of richness; Parcels = Diversity.

In relation to Jaccard's similarity indices (Table 2), the variation was 0.17 (Jatropha curcas, Croton heliotropiifolius, Tabebuia aurea, Clitorea ternatea, Capparis flexuosa L., Libidibia férrea, Pisonia tomentosa, Commiphora leptophloeos, Schinopsis brasiliensis, Eugenia pyriformis var. uvala, Ceiba glaziovii, Ziziphus joazeiro) to 0.83 (Mimosa Tenuiflora Wild).

In this sense, the similarity analyzed among the species studied can be considered low, except species 4 and 1, with similarity (Jac=83\%), followed by species 4 and 2, 4 and 3, 5 and 4 (Jac=80\%); 5 and 3, 6 and 2, 7 and 5, 8 and 5 (Jac=75\%); 2 and 1, 3 and 1, 5 and 1, 9 and 6, 9 and 7, 10 and 7, 10 and 8, 11 and 8 (Jac=67\%); and 3 and 2, 5 and 2, 7 and 4, 8 and 4 (Jac=60\%).

According to the data of figure 2, using the similarity index of Jaccard (Jac), the dendrogram was obtained for the sample species, through the formation of two distinct groups, with similarity cut around 0.5 of the Jaccard scale. This value of similarity was considered very low, since the Jaccard scale varies between the total dissimilarity ' 0 ' zero and the maximum similarity ' 1 ' one.

Table 2: Matrix of floristic similarity (Jaccard) of the species present in the study area.

\begin{tabular}{lllllllllllllllllllllllll}
\hline $\mathbf{p}$ & $\mathbf{1}$ & $\mathbf{2}$ & $\mathbf{3}$ & $\mathbf{4}$ & $\mathbf{5}$ & $\mathbf{6}$ & $\mathbf{7}$ & $\mathbf{8}$ & $\mathbf{9}$ & $\mathbf{1 0}$ & $\mathbf{1 1}$ & $\mathbf{1 2}$ & $\mathbf{1 3}$ & $\mathbf{1 4}$ & $\mathbf{1 5}$ & $\mathbf{1 6}$ & $\mathbf{1 7}$ & $\mathbf{1 8}$ & $\mathbf{1 9}$ & $\mathbf{2 0}$ & $\mathbf{2 1}$ & $\mathbf{2 2}$ & $\mathbf{2 3}$ \\
$\mathbf{p}$ & & & 0.6 & 0.6 & 0.8 & 0.6 & 0.5 & 0.5 & 0.5 & 0.3 & 0.3 & 0.3 & 0.1 & 0.1 & 0.1 & 0.1 & 0.1 & 0.1 & 0.1 & 0.1 & 0.1 & 0.1 & 0.1 & 0.1 \\
$\mathbf{1}$ & - & 7 & 7 & 3 & 7 & 0 & 0 & 0 & 3 & 3 & 3 & 7 & 7 & 7 & 7 & 7 & 7 & 7 & 7 & 7 & 7 & 7 & 7 \\
& & & 0.6 & 0.8 & 0.6 & 0.7 & 0.4 & 0.4 & 0.5 & 0.2 & 0.2 & 0.3 & 0.3 & 0.3 & 0.3 & 0.3 & 0.3 & 0.3 & 0.3 & 0.0 & 0.3 & 0.3 & 0.0 \\
$\mathbf{2}$ & - & - & 0 & 0 & 0 & 5 & 0 & 0 & 0 & 0 & 0 & 3 & 3 & 3 & 3 & 3 & 3 & 3 & 3 & 0 & 3 & 3 & 0 \\
& & & & 0.8 & 0.7 & 0.4 & 0.4 & 0.7 & 0.2 & 0.4 & 0.5 & 0.2 & 0.2 & 0.2 & 0.2 & 0.2 & 0.2 & 0.0 & 0.0 & 0.2 & 0.2 & 0.2 & 0.0 \\
$\mathbf{3}$ & - & - & - & 0 & 5 & 0 & 0 & 5 & 0 & 0 & 0 & 5 & 5 & 5 & 5 & 5 & 5 & 0 & 0 & 5 & 5 & 5 & 0 \\
& & & & & 0.8 & 0.6 & 0.6 & 0.6 & 0.4 & 0.4 & 0.4 & 0.2 & 0.2 & 0.2 & 0.2 & 0.2 & 0.2 & 0.2 & 0.2 & 0.2 & 0.2 & 0.2 & 0.0 \\
$\mathbf{4}$ & - & - & - & - & 0 & 0 & 0 & 0 & 0 & 0 & 0 & 0 & 0 & 0 & 0 & 0 & 0 & 0 & 0 & 0 & 0 & 0 & 0
\end{tabular}




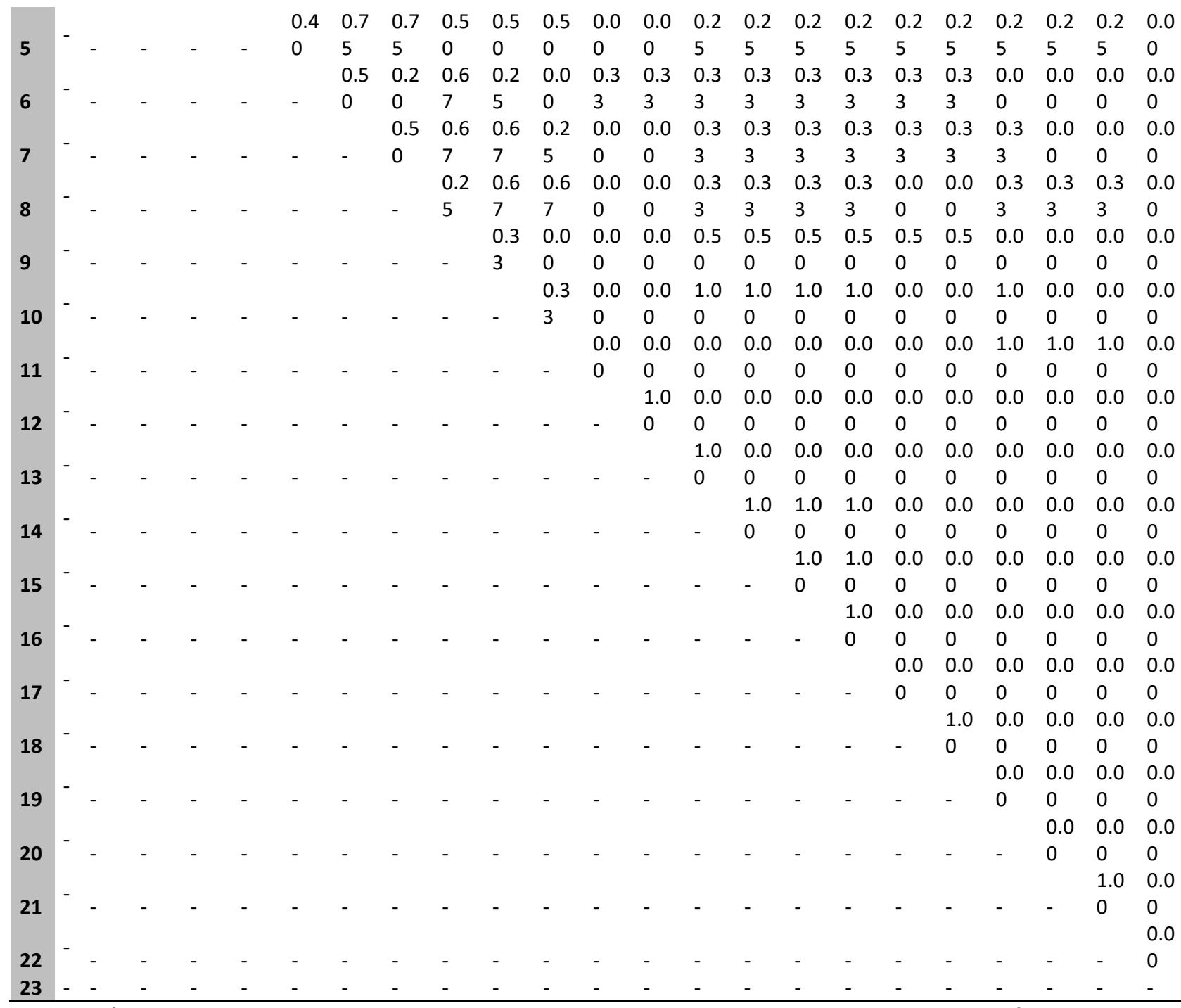

Note: The floristic species are represented by arabic numbers. $1=$ Croton sonderianus; $2=$ Aspidosperma pyrifolium; $3=$ Mimosa tenuiflora Wild; 4 = Piptdenia stipulacea Benth; 5 = Poincianella pyramidalis Tul.; 6 = Uncaria tomentosa; $7=$ Combretum glaucocarpum; 8 = Bauhinia forficata Linn; 9 = Acacia glomerosa Benth; $10=$ Capparis yco; $11=$ Manihot pseudoglaziovii; $12=$ Jatropha curcas; $13=$ Croton heliotropiifolius; $14=$ Tabebuia aurea; $15=$ Clitorea ternatea; $16=$ Capparis flexuosa L.; $17=$ Libidibia férrea; $18=$ Pisonia tomentosa; 19 = Commiphora leptophloeos; 20 = Schinopsis brasiliensis; $21=$ Eugenia pyriformis var. uvalha; $22=$ Ceiba glaziovii; 23 = Ziziphus joazeiro.

Regarding the phytosociological parameters of floristic remnants (Table 3), it was observed that the species Aspidosperma pyrifolium, Combretum glaucocarpum and Piptdenia stipulacea Benth, respectively, accounted for the largest basal area (AB), with $67.02 \%$ of the total basal area sampled. In relation to the highest absolute density (DA) and relative density (DR), the species Combretum glaucocarpum (1066.66 individuals / ha and 21.78\%), Croton sonderianus (833.33 individuals / ha and 17.86\%) and Aspidosperma pyrifolium (733.33 individuals / ha and $15.71 \%$ ).

For the absolute and relative frequency values, the species with the highest percentage were Croton sonderianus (100\% and 10.91\%), Mimosa Tenuiflora Wild (100\% and 10.91\%), Piptdenia stipulacea Benth (83.33\% and 9.09\%) and Aspidosperma pyrifolium (83.33\% and 9.09\%). In the analysis of absolute dominance (DoA) and relative dominance (DoR), Aspidosperma pyrifolium was the most expressive species (0.578 AB / ha and $36.82 \%$ ). The percentage of the coverage value (VC\%) and the percentage of importance value (VI\%) followed the same order, Aspidosperma pyrifolium and Combretum glaucocarpum. The species Combretum glaucocarpum, despite being the first in density, came in second place in importance value. 


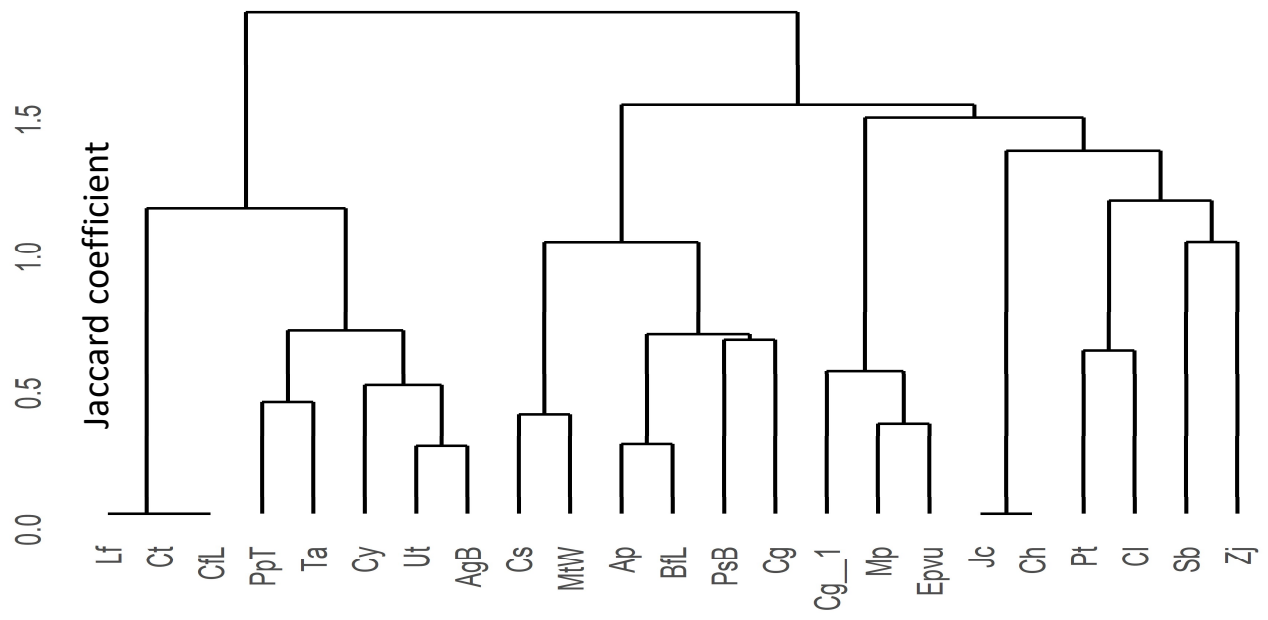

Figure 2: Dendrogram generated from the similarity index of Jaccard among the floristic species of the study area.

Note: The floristic species are represented by the initials. $\mathrm{Lf}=$ Libidibia férrea; $C t=$ Clitorea ternatea; $C f L=$ Capparis flexuosa L.; PpT $=$ Piptdenia stipulacea Benth; $\mathrm{Ta}=$ Tabebuia aurea; $\mathrm{Cy}=$ Capparis yco; $\mathrm{Ut}=$ Uncaria tomentosa; $\mathrm{AgB}=$ Acacia glomerosa Benth; $\mathrm{Cs}=$ Croton sonderianus; $\mathrm{MtW}=$ Mimosa tenuiflora Wild; $\mathrm{Ap}=$ Aspidosperma pyrifolium; $\mathrm{BfL}=$ Bauhinia forficata Linn; $\mathrm{PsB}=$ Poincianella pyramidalis Tul.; $\mathrm{Cg}=$ Ceiba glaziovii; $\mathrm{Cg} \_1=$ Combretum glaucocarpum; $\mathrm{Mp}=$ Manihot pseudoglaziovii; Epvu = Eugenia pyriformis var. uvalha; Jc = Jatropha curcas; $\mathrm{Ch}=$ Croton heliotropiifolius; $\mathrm{Pt}=$ Pisonia tomentosa; $\mathrm{Cl}=$ Commiphora leptophloeos; $\mathrm{Sb}=$ Schinopsis brasiliensis; $\mathrm{Zj}=$ Ziziphus joazeiro.

It should be noted that the same species (Mimosa Tenuiflora Wild) had the highest value: mean total height $(\mathrm{HT})$, with the mean height $=4.32 \mathrm{~m}$; standard deviation of total height $(8.04)$, and higher mean circumference at breast height (CAP). The values of the total height $(\mathrm{HT})$ variance were more expressive for the species Piptdenia stipulacea Benth $(3.48 \mathrm{~m})$ and the variance of the circumference at breast height (CAP) was higher in the species Poincianella pyramidalis Tul. $(37.74 \mathrm{~cm})$. This discrepancy in the circumference is justifiable, because there are species of different senescences in the study area, because the catingueira is a species that in the adult phase can reach more than $10 \mathrm{~m}$ in height and diameter of up to $50 \mathrm{~cm}$.

In relation to the Shannon-Weaver Diversity Index, there was variation in the plots, between 0.9 and 2.31, with larger diversity in plot 2, pointing to low diversity. The confidence interval of the Shannon-Weaver index, which ranged from 2.25 to 3.18, was also calculated by the Jackknife method. The Simpson's dominance index $(C)$ calculated in this study was also low $(C=0.57)$ and, far below $(C=1)$, thus demonstrating a low diversity in plot 6 .

Table 3: Phytosociological parameters of floristic remnants around Borborema Energética S.A.

\begin{tabular}{|c|c|c|c|c|c|c|c|c|c|c|c|c|c|c|c|c|c|}
\hline $\begin{array}{l}\text { Co } \\
\text { de }\end{array}$ & Scientific name & $\mathbf{N}$ & $\mathbf{U}$ & AB & DA & DR & FA & FR & $\begin{array}{l}\text { Do } \\
\text { A }\end{array}$ & $\begin{array}{l}\text { Do } \\
R\end{array}$ & $\begin{array}{l}\text { VC } \\
(\%)\end{array}$ & $\begin{array}{l}\text { VI } \\
(\%)\end{array}$ & $\begin{array}{l}\text { Averag } \\
\text { e HT }\end{array}$ & $\begin{array}{l}\text { Varianc } \\
\text { e HT }\end{array}$ & $\begin{array}{l}\text { Desv. } \\
\text { Pad HT }\end{array}$ & $\begin{array}{l}\text { Average } \\
\text { CAP }\end{array}$ & $\begin{array}{l}\text { Variance } \\
\text { CAP }\end{array}$ \\
\hline 1 & Croton sonderianus & 50 & 6 & $\begin{array}{l}0.0 \\
05\end{array}$ & $\begin{array}{l}833 . \\
33\end{array}$ & $\begin{array}{l}17 . \\
86\end{array}$ & 100 & $\begin{array}{l}10 . \\
91\end{array}$ & $\begin{array}{l}0.0 \\
89\end{array}$ & $\begin{array}{l}5.6 \\
7\end{array}$ & $\begin{array}{l}11.7 \\
6\end{array}$ & $\begin{array}{l}11 . \\
48\end{array}$ & 3.56 & 1.44 & 1.2 & 3.52 & 1.12 \\
\hline 2 & $\begin{array}{l}\text { Mimosa tenuiflora } \\
\text { Wild }\end{array}$ & 32 & 5 & $\begin{array}{l}0.0 \\
05\end{array}$ & $\begin{array}{l}533 . \\
33\end{array}$ & $\begin{array}{l}11 . \\
43\end{array}$ & 100 & $\begin{array}{l}10 . \\
91\end{array}$ & $\begin{array}{l}0.0 \\
85\end{array}$ & $\begin{array}{l}5.4 \\
3 \\
\end{array}$ & 8.43 & $\begin{array}{l}9.2 \\
6 \\
\end{array}$ & 10.67 & 1.78 & 8.04 & 14.95 & 1.39 \\
\hline 3 & $\begin{array}{l}\text { Piptdenia stipulacea } \\
\text { Benth }\end{array}$ & 23 & 5 & $\begin{array}{l}0.0 \\
1\end{array}$ & 350 & 7.5 & $\begin{array}{l}83.3 \\
3 \\
\end{array}$ & $\begin{array}{l}9.0 \\
9\end{array}$ & $\begin{array}{l}0.1 \\
65\end{array}$ & $\begin{array}{l}10 . \\
53\end{array}$ & 9.01 & $\begin{array}{l}9.0 \\
4\end{array}$ & 4.43 & 3.48 & 1.87 & 5.87 & 26.14 \\
\hline 4 & Jatropha curcas & 3 & 1 & 0 & $\begin{array}{l}16.6 \\
6\end{array}$ & $\begin{array}{l}0.3 \\
6\end{array}$ & $\begin{array}{l}16.6 \\
7\end{array}$ & $\begin{array}{l}1.8 \\
2\end{array}$ & $\begin{array}{l}0.0 \\
01\end{array}$ & $\begin{array}{l}0.0 \\
8\end{array}$ & 0.22 & $\begin{array}{l}0.7 \\
5\end{array}$ & 1.8 & 0 & 1.8 & 3.1 & 0 \\
\hline 5 & $\begin{array}{l}\text { Aspidosperma } \\
\text { pyrifolium }\end{array}$ & 44 & 5 & $\begin{array}{l}0.0 \\
35\end{array}$ & $\begin{array}{l}733 . \\
33\end{array}$ & $\begin{array}{l}15 . \\
71\end{array}$ & $\begin{array}{l}83.3 \\
3 \\
\end{array}$ & $\begin{array}{l}9.0 \\
9 \\
\end{array}$ & $\begin{array}{l}0.5 \\
78\end{array}$ & $\begin{array}{l}36 . \\
82\end{array}$ & $\begin{array}{l}26.2 \\
7\end{array}$ & $\begin{array}{l}20 . \\
54\end{array}$ & 4.83 & 1.31 & 1.14 & 7.63 & 41.9 \\
\hline 6 & Uncaria tomentosa & 5 & 3 & $\begin{array}{l}0.0 \\
02\end{array}$ & $\begin{array}{l}83.3 \\
3 \\
\end{array}$ & $\begin{array}{l}1.7 \\
9\end{array}$ & 50 & $\begin{array}{l}5.4 \\
5 \\
\end{array}$ & $\begin{array}{l}0.0 \\
29\end{array}$ & $\begin{array}{l}1.8 \\
4\end{array}$ & 1.81 & $\begin{array}{l}3.0 \\
3\end{array}$ & 5.16 & 2.6 & 1.61 & 6.22 & 6.04 \\
\hline 7 & $\begin{array}{l}\text { Croton } \\
\text { heliotropiifolius }\end{array}$ & 3 & 1 & 0 & 50 & $\begin{array}{l}1.0 \\
7\end{array}$ & $\begin{array}{l}16.6 \\
7\end{array}$ & $\begin{array}{l}1.8 \\
2\end{array}$ & $\begin{array}{l}0.0 \\
04\end{array}$ & $\begin{array}{l}0.2 \\
3\end{array}$ & 0.65 & $\begin{array}{l}1.0 \\
4\end{array}$ & 2.4 & 0 & 0 & 3 & 0 \\
\hline 8 & Tabebuia aurea & 1 & 1 & 0 & $\begin{array}{l}16.6 \\
6\end{array}$ & $\begin{array}{l}0.3 \\
6 \\
\end{array}$ & $\begin{array}{l}16.6 \\
7 \\
\end{array}$ & $\begin{array}{l}1.8 \\
2 \\
\end{array}$ & $\begin{array}{l}0.0 \\
02 \\
\end{array}$ & $\begin{array}{l}0.1 \\
3 \\
\end{array}$ & 0.25 & $\begin{array}{l}0.7 \\
7\end{array}$ & 4 & 0 & 4 & 4 & 0 \\
\hline 9 & Capparis yco & 3 & 2 & 0 & 50 & $\begin{array}{l}1.0 \\
7\end{array}$ & $\begin{array}{l}33.3 \\
3\end{array}$ & $\begin{array}{l}3.6 \\
4 \\
\end{array}$ & $\begin{array}{l}0.0 \\
01\end{array}$ & $\begin{array}{l}0.0 \\
9\end{array}$ & 0.58 & 1.6 & 1.33 & 1.61 & 1.27 & 1.67 & 1.33 \\
\hline 10 & $\begin{array}{l}\text { Bauhinia forficata } \\
\text { Linn }\end{array}$ & 25 & 3 & $\begin{array}{l}0.0 \\
05\end{array}$ & $\begin{array}{l}416 . \\
66\end{array}$ & $\begin{array}{l}8.9 \\
3\end{array}$ & 50 & $\begin{array}{l}5.4 \\
5\end{array}$ & $\begin{array}{l}0.0 \\
82\end{array}$ & $\begin{array}{l}5.2 \\
2\end{array}$ & 7.07 & $\begin{array}{l}6.5 \\
3\end{array}$ & 4.42 & 0.26 & 0.51 & 4.6 & 3.77 \\
\hline
\end{tabular}




\begin{tabular}{|c|c|c|c|c|c|c|c|c|c|c|c|c|c|c|c|c|c|}
\hline 11 & $\begin{array}{l}\text { Combretum } \\
\text { glaucocarpum }\end{array}$ & 61 & 3 & $\begin{array}{l}0.0 \\
18 \\
\end{array}$ & $\begin{array}{l}1016 \\
.66 \\
\end{array}$ & $\begin{array}{l}21 . \\
78\end{array}$ & $\begin{array}{l}66.6 \\
7 \\
\end{array}$ & $\begin{array}{l}7.2 \\
7 \\
\end{array}$ & 0.3 & $\begin{array}{l}19 . \\
09\end{array}$ & $\begin{array}{l}20.4 \\
4 \\
\end{array}$ & $\begin{array}{l}16 . \\
05\end{array}$ & 6.24 & 0.81 & 0.9 & 8.63 & 9.45 \\
\hline 12 & Clitorea ternatea & 1 & 1 & 0 & $\begin{array}{l}16.6 \\
6\end{array}$ & $\begin{array}{l}0.3 \\
6\end{array}$ & $\begin{array}{l}16.6 \\
7\end{array}$ & $\begin{array}{l}1.8 \\
2\end{array}$ & $\begin{array}{l}0.0 \\
01\end{array}$ & $\begin{array}{l}0.0 \\
8\end{array}$ & 0.22 & $\begin{array}{l}0.7 \\
5\end{array}$ & 4.5 & 0 & 4.5 & 3 & 0 \\
\hline 13 & $\begin{array}{l}\text { Poincianella } \\
\text { pyramidalis Tul. }\end{array}$ & 12 & 4 & $\begin{array}{l}0.0 \\
08\end{array}$ & 200 & $\begin{array}{l}4.2 \\
9 \\
\end{array}$ & $\begin{array}{l}66.6 \\
7 \\
\end{array}$ & $\begin{array}{l}7.2 \\
7 \\
\end{array}$ & $\begin{array}{l}0.1 \\
41 \\
\end{array}$ & $\begin{array}{l}9.0 \\
1 \\
\end{array}$ & 6.65 & $\begin{array}{l}6.8 \\
6 \\
\end{array}$ & 5.04 & 0.48 & 0.69 & 7.37 & 37.74 \\
\hline 14 & Capparis flexuosa $L$ & 1 & 1 & 0 & $\begin{array}{l}16.6 \\
6 \\
\end{array}$ & $\begin{array}{l}0.3 \\
6 \\
\end{array}$ & $\begin{array}{l}16.6 \\
7 \\
\end{array}$ & $\begin{array}{l}1.8 \\
2 \\
\end{array}$ & $\begin{array}{l}0.0 \\
01\end{array}$ & $\begin{array}{l}0.0 \\
3 \\
\end{array}$ & 0.2 & $\begin{array}{l}0.7 \\
4\end{array}$ & 3.5 & 0 & 3.5 & 2 & 0 \\
\hline 15 & $\begin{array}{l}\text { Acacia glomerosa } \\
\text { Benth }\end{array}$ & 2 & 2 & 0 & $\begin{array}{l}33.3 \\
3 \\
\end{array}$ & $\begin{array}{l}0.7 \\
1\end{array}$ & $\begin{array}{l}33.3 \\
3 \\
\end{array}$ & $\begin{array}{l}3.6 \\
4 \\
\end{array}$ & $\begin{array}{l}0.0 \\
06\end{array}$ & $\begin{array}{l}0.4 \\
1 \\
\end{array}$ & 0.56 & $\begin{array}{l}1.5 \\
9\end{array}$ & 5 & 2 & 1.41 & 4.75 & 3.13 \\
\hline 16 & Libidibia ferrea & 1 & 1 & 0 & $\begin{array}{l}16.6 \\
6\end{array}$ & $\begin{array}{l}0.3 \\
6\end{array}$ & $\begin{array}{l}16.6 \\
7\end{array}$ & $\begin{array}{l}1.8 \\
2\end{array}$ & $\begin{array}{l}0.0 \\
02\end{array}$ & $\begin{array}{l}0.1 \\
3\end{array}$ & 0.25 & $\begin{array}{l}0.7 \\
7\end{array}$ & 5.5 & 0 & 5.5 & 4 & 0 \\
\hline 17 & Pisonia tomentosa & 1 & 1 & 0 & $\begin{array}{l}16.6 \\
6\end{array}$ & $\begin{array}{l}0.3 \\
6\end{array}$ & $\begin{array}{l}16.6 \\
7\end{array}$ & $\begin{array}{l}1.8 \\
2\end{array}$ & $\begin{array}{l}0.0 \\
06\end{array}$ & $\begin{array}{l}0.3 \\
6\end{array}$ & 0.36 & $\begin{array}{l}0.8 \\
4\end{array}$ & 5.5 & 0 & 5.5 & 6.5 & 0 \\
\hline 18 & $\begin{array}{l}\text { Commiphora } \\
\text { leptophloeos }\end{array}$ & 3 & 1 & $\begin{array}{l}0.0 \\
02\end{array}$ & 50 & $\begin{array}{l}1.0 \\
7\end{array}$ & $\begin{array}{l}16.6 \\
7\end{array}$ & $\begin{array}{l}1.8 \\
2\end{array}$ & $\begin{array}{l}0.0 \\
26\end{array}$ & $\begin{array}{l}1.6 \\
3\end{array}$ & 1.35 & $\begin{array}{l}1.5 \\
1\end{array}$ & 5 & 3 & 1.73 & 7.2 & 18.72 \\
\hline 19 & $\begin{array}{l}\text { Manihot } \\
\text { pseudoglaziovii }\end{array}$ & 4 & 2 & $\begin{array}{l}0.0 \\
01\end{array}$ & $\begin{array}{l}66.6 \\
6\end{array}$ & $\begin{array}{l}1.4 \\
3\end{array}$ & $\begin{array}{l}33.3 \\
3\end{array}$ & $\begin{array}{l}3.6 \\
4\end{array}$ & $\begin{array}{l}0.0 \\
14\end{array}$ & $\begin{array}{l}0.8 \\
8\end{array}$ & 1.15 & $\begin{array}{l}1.9 \\
8\end{array}$ & 4.88 & 0.73 & 0.85 & 5 & 1.33 \\
\hline 20 & $\begin{array}{l}\text { Schinopsis } \\
\text { brasiliensis }\end{array}$ & 1 & 1 & 0 & $\begin{array}{l}16.6 \\
6 \\
\end{array}$ & $\begin{array}{l}0.3 \\
6 \\
\end{array}$ & $\begin{array}{l}16.6 \\
7 \\
\end{array}$ & $\begin{array}{l}1.8 \\
2 \\
\end{array}$ & $\begin{array}{l}0.0 \\
07 \\
\end{array}$ & $\begin{array}{l}0.4 \\
1 \\
\end{array}$ & 0.39 & $\begin{array}{l}0.8 \\
6 \\
\end{array}$ & 8 & 0 & 8 & 7 & 0 \\
\hline 21 & $\begin{array}{l}\text { Eugenia pyriformis } \\
\text { var. uvalha }\end{array}$ & 2 & 1 & 0 & $\begin{array}{l}33.3 \\
3 \\
\end{array}$ & $\begin{array}{l}0.7 \\
1 \\
\end{array}$ & $\begin{array}{l}16.6 \\
7 \\
\end{array}$ & $\begin{array}{l}1.8 \\
2 \\
\end{array}$ & $\begin{array}{l}0.0 \\
06 \\
\end{array}$ & $\begin{array}{l}0.3 \\
8 \\
\end{array}$ & 0.55 & $\begin{array}{l}0.9 \\
7 \\
\end{array}$ & 4.25 & 0.13 & 0.35 & 4.5 & 4.5 \\
\hline 22 & Ceiba glaziovii & 1 & 1 & 0 & $\begin{array}{l}16.6 \\
6\end{array}$ & $\begin{array}{l}0.3 \\
6\end{array}$ & $\begin{array}{l}16.6 \\
7\end{array}$ & $\begin{array}{l}1.8 \\
2\end{array}$ & $\begin{array}{l}0.0 \\
01\end{array}$ & $\begin{array}{l}0.0 \\
8\end{array}$ & 0.22 & $\begin{array}{l}0.7 \\
5\end{array}$ & 4 & 0 & 4 & 3 & 0 \\
\hline \multirow[t]{2}{*}{23} & Ziziphus joazeiro & 3 & 1 & $\begin{array}{l}0.0 \\
01 \\
\end{array}$ & 50 & $\begin{array}{l}1.0 \\
7 \\
\end{array}$ & $\begin{array}{l}16.6 \\
7 \\
\end{array}$ & $\begin{array}{l}1.8 \\
2 \\
\end{array}$ & $\begin{array}{l}0.0 \\
1 \\
\end{array}$ & $\begin{array}{l}0.6 \\
3 \\
\end{array}$ & 0.85 & $\begin{array}{l}1.1 \\
7 \\
\end{array}$ & 4 & 0 & 0 & 5 & 0 \\
\hline & $* * *$ Total & $\begin{array}{l}28 \\
0\end{array}$ & 6 & $\begin{array}{l}0.0 \\
94\end{array}$ & $\begin{array}{l}4666 \\
.66\end{array}$ & 100 & $\begin{array}{l}916 . \\
67\end{array}$ & 100 & $\begin{array}{l}1.5 \\
71\end{array}$ & 100 & 100 & 100 & 4.32 & 1.11 & 2.21 & 4.98 & 137.1 \\
\hline
\end{tabular}

Legend: $\mathbf{N}=$ Number of trees; $\mathbf{U}=$ Number of sample units in which the species occurs; $\mathrm{AB}=\mathrm{Basal}$ area; $\mathbf{D A}=\mathrm{Absolute}$ Density (N/ha); $\mathbf{D R}=$ Relative Density; FA = Absolute Frequency; FR = Relative Frequency; Doa = Absolute Dominance $(A B /$ ha); DoR = Relative Dominance; $\mathbf{V C}(\%)$ = Percentage of Coverage Amount; VI = Value of Importance; VI(\%) = Percentage of Importance Value; HT = Total Height $(\mathrm{m}) ; \mathbf{C A P}=$ Circumference at Breast Height $(\mathrm{cm})$.

Regarding the Pielou equation value $(\mathrm{J})$, the result was 0.76 , indicating that $76 \%$ of theoretical maximum diversity was obtained by means of the sampling performed. For the Jentsch Coefficient of Mixing (QM), the ratio 1:12.1 was found, indicating an average of 12 individuals for each species sampled. Therefore, the low diversity is ratified for the population presented, because the value of QM was far from the value 1.

In this sense, it is verified that the low floristic diversity of the caatinga is generally linked to the natural limitations of the region, such as shallow soils, semi - aridity and marked anthropization, culminating in the predominance of a few generalist taxa and in the heterogeneous distribution of abundance of individuals, with a marked presence of primary and secondary succession plants.

\section{DISCUSSION}

The area under study is in the process of ecological succession, due to the physical pressure of the surrounding residents, who exploit the natural resources of the area in an uncontrolled way, thus causing deforestation, burning, presence of clearings, and a consequent reduction of local biodiversity. As for the number of families recorded in the study, the data are similar to the data of Pereira Júnior et al. (2014). In the 30-hectare area, 16 families were identified, with Fabaceae ( $n=12)$, Anacardiaceae and Euphorbiaceae, both with 4 representatives.

The higher number of individuals of the species Combretum glaucocarpum recorded in plots 2, 3, 4 is justifiable, because these plots are located within the vegetative remnants that make up the study area. The occurrence of this vegetation is common in the semiarid areas of the Brazilian Northeast, because it is characterized as arboreal caatinga and, as an adult, can reach up to about 12 meters. The greater diversity 
of Croton sonderianus is also attributed to it being an area modified by anthropic actions, and this plant is present in the initial or intermediate stages of ecological succession and natural regeneration.

It is also worth noting that the low diversity of the studied area is a characteristic feature of the caatinga biome, when compared to other physiognomies, mainly of other Brazilian biomes, since this situation is made possible by being more exposed to the anthropic disturbances. With this, further damaging the ecological integrity of the region, which mainly affects biodiversity and people's quality of life.

On the Jaccard index, which is widely used to compare floristic species and to determine the similarity of plots in terms of species composition, it was found that the similarity is maximal when the value is equal to one, and, non-existent, when it is zero. For indexes above 0.5, similarity is high (FERREIRA JÚNIOR et al., 2008). As for similarity, our data differ from Rabbani et al. (2012), because, using the same methodological procedure, in 18 varieties of Genipa americana L, in an area of riparian forest in the Lower Course of the São Francisco Sergipano River, (G1 and G12, G8 and G2, G7 and G5, G7 and G5, G7 and G8, G9 and G8, and G11 and G10).

Comparing the density indices, which are important factors to be considered in forest inventories, Araújo et al. (2012), in a study in the Paraiba caatinga, municipality of Santa Luzia-PB, registered high expressiveness for density values in the species Mimosa hostilis, with $79.18 \%$ and, Cnidoscolus phyllacanthus with $14.52 \%$, totaling $93.70 \%$ of the total value of relative density, thus confirming a high degree of degradation in that area, since these species generally stand out in the initial and secondary occupation of degraded areas.

A trend similar to these results is followed in the phytosociological records by Marangon et al. (2013), in an area of Cariri Paraíba, where the species with the highest relative density were Croton rhamnifolius (27.12\%), Poincianella bracteosa (24.06\%), Mimosa ophtalmocentra (11.11\%) and Manihot cartagenas subsp. glaziovii (9.58\%), which contributed more than $70 \%$ in relation to this parameter in the study area. Given this, few species are observed, as in other studies in different caatinga physiognomies, demonstrating more than half of the importance related to the study area, such as Bessa et al. (2011), Calixto Júnior et al. (2014), Guedes et al. (2012) and Leitão et al. (2014).

Confronting the results of this research regarding the richness and diversity indexes estimated by the Shannon index $\left(\mathrm{H}^{\prime}\right)$ for the study area, the data of Guedes et al. (2012) were similar in a study carried out with a forest reserve at Fazenda Tamanduá, Santa Terezinha, Paraíba, Brazil, where the values of 2.54, for the Shannon index $\left(\mathrm{H}^{\prime}\right), 0.96$ for Simpson's dominance $(\mathrm{C})$ and 0.82 for Pielou equitability $(\mathrm{J})$ were obtained.

The results of Alves Junior et al. (2013), also show similarity to this study, where a forest inventory was carried out in caatinga vegetation, at Fazenda Itapemirim, in the municipality of Floresta-PE, cataloging 15 botanical species, totaling 208 individuals, presenting Shannon-Weaver diversity index $\left(\mathrm{H}^{\prime}\right)$, with a value of 1.91, Pielou uniformity index of 0.705 , Simpson's dominance index of 0.771 , and Jentsch Mixing Coefficient (QM) equal to 0.072 . 


\section{CONCLUSIONS}

It was observed that the families Euphorbiaceae and Fabaceae were the most representative of arboreal shrub species, which confirms the trend of other studies related to the Brazilian semiarid regions. The species Croton sonderianus and Mimosa tenuiflora Wild were recorded in the majority of the plots and presented high numbers of individuals in relation to the other cataloged species and were registered principally at the edges of the vegetation remnants inventoried. It was also verified that all diversity verification indexes had low values, in addition to the species that also had low richness.

Regarding the ecological integrity of the arboreal remnants of the Thermoelectric Borborema Energética SA, there are indications of degradation, anthropization and / or recovery of this area, which has already been altered and is in the process of recovering the original structure. Also, the fact that the studied floristic composition presents low values regarding height and circumference can be attributed to the low age of the shrub tree species. Thus, floristic inventories are of great importance to monitor biodiversity, as well as to set goals and establish efficient environmental management actions, serving as a tool for the conservation of caatinga species and as a benchmark for comparison with future inventories.

\section{REFERENCES}

BESSA, M. A. P.; MEDEIROS, J. F.. Levantamento florístico e fitossociológico em fragmentos de caatinga no município de Tabuleiro Grande/RN. Geo Temas, Pau dos Ferros, v.1, n.2, p.69-83, 2011.

BRAUN-BLANQUET, J.. Fitosociología: Bases para el estudio de las comunidades vegetales. Madrid: H. Blume, 1979.

BRUMMITT, R. K.; POWELL, C. E.. Authors of plant names: A list of authors of scientific names of plants, with recommended standard form of their names including abbreviations. Royal Botanic Gardens: Kew, 1992.

CALIXTO JÚNIOR, J. T.; DRUMOND, M. A.. Estudo comparativo da estrutura fitossociológica de dois fragmentos de Caatinga em níveis diferentes de conservação. Pesq. flor. bras., Colombo, v.34, n.80, p.00-00, 2014.

CEN, S.. Biological Monitoring of Air Pollutants and Its Influence on Human Beings. Open Biomed Eng. J., v.9, n.1, p.219-223, 2015. DOI: http://10.2174/1874120701509010219

CIENTEC. Consultoria e Desenvolvimento de Sistemas. Software. Mata Nativa versão 4.1.0.14. Viçosa, 2016.

FERREIRA JÚNIOR, E. V.; SOARES, T. S.; COSTA, M. F. F.; SILVA, V. S. M.. Composição, diversidade e similaridade florística de uma floresta tropical semidecídua submontana em Marcelândia/MT. Acta Amazônica, v.38, n.4, p.673-680. 2008.

GIULIETTI, A. M.; BOCAGE NETA, A. L.; CASTRO, A. A. J. F.; GAMARRA-ROJAS, C. F. L.; SAMPAIO, E. V. S. B.; VIRGÍNIO, J. F.; QUEIROZ, L. P.; FIGUEIREDO, M. A.; RODAL, M. J. N.; BARBOSA, M. R. V.; HARLEY, R. M.. Diagnóstico da vegetação nativa do bioma Caatinga: Biodiversidade da
Caatinga: áreas e ações prioritárias para a conservação. 2004.

GUEDES, R. S.; ZANELLA, F. C. V.; COSTA JÚNIOR, J. E. V.; SANTANA, G. M.; SILVA, J. A.. Caracterização florísticofitossociológica do componente lenhoso de um trecho de Caatinga no semiárido Paraibano. Revista Caatinga, v.25, n.2, p.99-108, 2012.

KENT, M.; COKER, P.. Vegetation description and analysis a pratical approach. Chichester: John Wiley and Sons, 1989.

LEITÃO, A. C.; VASCONCELOS, W. A.; CAVALCANTE, A. M. B.; TINÔCO, L. B. M.; FRAGA, V. S.. Florística e estrutura de um ambiente transicional caatinga mata atlântica. Revista Caatinga, v.27, n.3, p.200-210, 2014.

MARANGON, G. P.; FERREIRA, R. L. C.; SILVA, J. A. A.; SOUZA, D. F.; SILVA, E. A.; LOUREIRO, G. H.. Estrutura e padrão espacial da vegetação em uma área de caatinga. Floresta, v.43, n.1, p.83-92, 2013.

MORAES, W. B.; JESUS JUNIOR, W. C.; BELAN, L. L.; PEIXOTO, L. A.; MORAES, W. B.; CECÍLIO, R. A.. Mudanças climáticas e seus potenciais impactos sobre os métodos de manejo de doenças de planta. Nucleus, v.8, n.1, p.2011. DOI: http://dx.doi.org/10.3738/nucleus.v8i1.555

MORO, M. F.; MARTINS, F. R.. Métodos de Levantamento do Componente Arbóreo-Arbustivo. In: FELFILI, J. M.; EISENLOHR, P. V.; MELO, M. M. R. F.; ANDRADE, L. A.; MEIRA NETO, J. A. A.. Fitossociologia no Brasil: Métodos e Estudos de Casos, Comissão de Especialistas em Fitossociologia/Sociedade Botânica do Brasil. UFV, 2011.

MUELLER-DOMBOIS, D.; ELLENBERG, H.. Aims and methods of vegetation Ecology. New York: John Wiley and Sons, 1974. 
OLIVEIRA, J. A.; GONÇALVES, P. R.; BONVICINO, C. R.. Mamíferos da Caatinga. In: LEAL, I. R.; TABARELLI, M.; SILVA, J. M. C.. Ecologia e Conservação da Caatinga. 2 ed. Recife: Universitária UFPE, 2003. p.822.

OLIVEIRA, L. T.; FERREIRA, M. Z.; CARVALHO, L. M. T.; FERRAZ FILHO, A. C.; OLIVEIRA, T. C. A.; SILVEIRA, E. M. O.; ACERBI JUNIOR, W. F.. Determinação do volume de madeira em povoamento de eucalipto por escâner a laser aerotransportado. Pesq. agropec. bras., v.49, n.9, p.692699, 2014. DOI: http://dx.doi.org10.1590/S0100$\underline{204 \times 2014000900005}$

PATRA, B.; DEY, S. K.; DAS, M. T.. Forest Management Practices for Conservation of Biodiversity: An Indian Perspective. International Journal of Environmental Biology, v.5, n.4, p.93-98, 2015.

PEREIRA JÚNIOR, L. R.; ANDRADE, A. P. D.; ARAÚJO, K. D.; BARBOSA, A. D. S.; BARBOSA, F. M.. Espécies da caatinga como alternativa para o desenvolvimento de novos fitofármacos. Floresta e Ambiente, v.21, n.4, p.509-520, 2014. DOI: http://dx.doi.org/10.1590/2179-8087.024212

RABBANI, A. R. C.; SILVA-MANN, R.; FERREIRA, R. A.. Variabilidade genética de Genipa americana L. pertencente ao baixo curso do Rio São Francisco. Revista Árvore, v.36, n.3, p.401-409, 2012. DOI: http://dx.doi.org/10.1590/S0100$\underline{67622012000300002}$

RODAL, M. J. N.; NASCIMENTO, L. M.. The Arboreal Component of a Dry Forestin Northeastern Brazil. Braz. J. Biol., v.66, n.2, p.479-491, 2006

SANTOS, H. G.; JACOMINE, P. K. T.; ANJOS, L. H. C.; OLIVEIRA, V. A.; OLIVEIRA, J. D.; COELHO, M. R.; LUMBRERAS, J. F.; CUNHA, T. J. F.. Sistema brasileiro de classificação de solos. Embrapa Solos-Livros técnicos (INFOTECA-E), 2006.

SNEATH, P. H.; SOKAL, R. R.. Numerical taxonomy the principles and practice of numerical classification. San Francisco: W. H. Freeman, 2006.

SOUZA, B. I.; ARTIGAS, R. C.; LIMA, E. R. V.. Caatinga e desertificação. Mercator, v.14, n.1, p.131-150, 2015. DOI: http://dx.doi.org/10.4215/RM2015.1401.0009

TARDIEU, F.. Plant response to environmental conditions: assessing potential production, water demand, and negative effects of water déficit. Front Physiol., v.4, n.17, 2013. DOI: http://10.3389/fphys.2013.00017

A CBPC - Companhia Brasileira de Produção Científica (CNPJ: 11.221.422/0001-03) detém os direitos materiais desta publicação. Os direitos referem-se à publicação do trabalho em qualquer parte do mundo, incluindo os direitos às renovações, expansões e disseminações da contribuição, bem como outros direitos subsidiários. Todos os trabalhos publicados eletronicamente poderão posteriormente ser publicados em coletâneas impressas sob coordenação da Sustenere Publishing, da Companhia Brasileira de Produção Científica e seus parceiros autorizados. Os (as) autores (as) preservam os direitos autorais, mas não têm permissão para a publicação da contribuição em outro meio, impresso ou digital, em português ou em tradução. 